\title{
Principle component analysis of exposure variation analysis during computer work at presence of delayed onset muscle soreness
}

\author{
A. Samani ${ }^{\mathrm{a},{ }^{*}}$, A. Kawczyński ${ }^{\mathrm{b}}, \mathrm{Chmura} \mathrm{J}^{\mathrm{b}}$ and P. Madeleine ${ }^{\mathrm{b}}$ \\ ${ }^{a}$ Center for Sensory-Motor Interaction (SMI), Department of Health Science and Technology, Aalborg University, \\ Aalborg, Denmark \\ ${ }^{\mathrm{b}}$ Depatment of Athletes Motor Skills, Sport Institute, University School of Physical Education, Wroclaw, Poland
}

\begin{abstract}
Eleven healthy volunteers took part in three sessions where computer work was performed for 10 min in each session. The recordings were made on two consecutive days. After the first session, the subjects underwent a series of high intensity eccentric exercises of shoulder elevation to induce muscle soreness. The second session was performed immediately after the exercises and third session 24 hours after the exercise. Surface electromyography (EMG) of descending and ascending trapezius, deltoideus anterior and serratus anterior was recorded. Exposure variation analysis (EVA) centroid and marginal distribution was computed. Principle component analysis (PCA) was performed on both cases. Describing more than $80 \%$ of variance, first eight components for EVA marginal distributions and first four ones for EVA centroid were picked to be investigated. The sole EVA amplitude levels played a practically significant role to describe the variation in EVA marginal distribution. However, a significant pattern of load redistribution to superficial muscles was not found. This may suggest investigating the role of deep muscles in redistribution of load during computer work in response to high level eccentric contractions.
\end{abstract}

Keywords: eccentric exercises; spatial reorganization; fatigue

\section{Introduction}

In response to altered sensory information due to e.g. muscle fatigue or experimental pain, a reorganization of muscular activity pattern in trapezius has been reported in several studies [2, 8]. Particularly, an increase of muscular load has been observed in the ascending trapezius, anterior deltoid and serratus anterior when descending trapezius rendered the source of biofeedback [13]. We have limited knowledge concerning muscle soreness and motor control interaction in the shoulder girdle muscles during precision tasks like computer work.

Delayed onset muscle soreness (DOMS) can be induced by performing bouts of excessive eccentric exercises in an experimental setup. This furnishes a reliable framework to study some aspects of the muscle pain and musculoskeletal disorders $[12,17,19]$. Inducing DOMS in an experimental setup may be reminiscent of different phases of the muscle damage [3] which may cause changes in the pattern of muscle activity and/or redistribution of physical load to other synergistic muscles [16].

If the processing of electromyographic (EMG) signals can discriminate between different phases of mimicked muscle damage, a designing framework for a biofeedback system can be established [14]. The system alerts a warning signal, for example, when the pattern of muscle activity gets close to that of damaged muscles during computer work.

Exposure variation analysis (EVA) presents a thorough estimation of physical exposure in occupational setups [11]. However, different methods have been suggested to extract relevant information from EVA $[4,15]$. Principle component analysis is a mathematical tool to extract a reduced space of a few uncorrelated factors from multiple correlated variables. The first principal component accounts for as much of the

*Corresponding author. E-mail: afsamani@hst.aau.dk 
variability in the data as possible and each succeeding component accounts for as much of the remaining variability as possible. As a constraint, all of these components get to be mutually orthogonal. Therefore, PCA can be utilized to reduce information from EVA in an optimal way.

In the current study, we investigated the changes in the pattern of muscle activity in presence of DOMS and compare it to the baseline. We hypothesized that there would be a systematic redistribution of workload in presence of DOMS.

\section{Methods and subjects}

\subsection{Subjects}

Eleven male subjects (aged 25.3 \pm 5.0 years; height $177.9 \pm 5.8 \mathrm{~cm}$; body mass $69.8 \pm 6.4 \mathrm{~kg}$, BMI $22.0 \pm 1.3$ $\mathrm{kg} / \mathrm{m} 2$ ) participated in the present study. Informed consent was obtained from each subject. All participants reported no pain in the shoulder region prior to the experiment and had no history of neck-shoulder disorders. All subjects maintained normal daily activity during the course of the study. None had participated in strength training in the past month. The study was approved by the local ethics committee (No. N-20070004) and conducted in accordance with the Declaration of Helsinki.

\subsection{Experimental protocol}

The experiment was performed over two consecutive days. The procedure composed of computer work performed before, immediately after and 24 hours after eccentric exercises. These three time instances were named here as sessions. Once the subjects had received instructions and surface electromyogram (SEMG) electrodes were placed (see below). The following steps were sequentially repeated before (session one), immediately after (session two) and 24 hours after (session three) eccentric exercises aiming at assessing muscle activation in respectively, a fresh, fatigued and sore trapezius muscle.

\subsubsection{Resting contraction}

The resting level of EMG was calculated from 1 min of instructed rest with both hands on the laps. The period of $5 \mathrm{~s}$ with lowest RMS value during the instructed rest was quadratically subtracted as defined in [1] from all other EMG signals.

\subsubsection{Reference contraction}

The subjects were seated upright on a comfortable chair with their palms towards the ground looking straight forward. In this position, they were asked to perform one reference contraction consisting of bilateral arm abduction at $90^{\circ}$ in the frontal plane for $5 \mathrm{~s}$ [10].

\subsubsection{Computer work}

Ten minutes of standardized computer work was performed [15]. It consists of duplicating various graphs showing six circular targets linked to each other by straight lines including a start target (bold circle) displayed on the upper right corner of a computer screen (pixel resolution: $0.3 \mathrm{~mm}$, screen resolution: $1024 \times 768$ pixels). Once the two graphs were identical, a new graph appeared. The time allowed for completing a graph was 12 seconds at low work pace, and eight seconds for the high work pace.

\subsection{Eccentric exercises}

During each eccentric contraction subject had to counteract the vertical force exerted by a dynamometer as much as possible over previously defined shoulder range of motion. The subject raised both shoulders and wearing a corset during the exercise to avoid lateral bending [9].

The eccentric exercise protocol used to induce DOMS and consisted of 50 eccentric contractions of right shoulder which was divided into 5 bouts including 10 contractions at maximum voluntary contraction level and the bouts were separate by 2 min resting period. The maximum voluntary contraction was measured under the same dynamometer right before the exercises.

\subsection{Data recording and processing}

EMG was collected from four sites, namely, descending and ascending parts of trapezius muscle, deltoideus anterior and serratus anterior. Bipolar surface electrodes were aligned (inter-electrodes distance of $2 \mathrm{~cm}$ ) along the direction of the muscle fibers. Electrodes were placed (i) $\sim 20 \%$ lateral to the midpoint between the acromion and the $\mathrm{C} 7$ vertebra for the descending part, (ii) $\sim 33 \%$ medial to the midpoint between the medial scapular and the T8 vertebra for the ascending part (iii) within the elongated oval area $2 \mathrm{~cm}$ below the lateral end of the clavicle for deltoid anterior (iv) on the fourth rib from the top on the midaxillary line for serratus anterior. The ref- 
erence electrode was placed over the C7 spinal process. The EMG signals were amplified 2000 times, and band pass filtered [5-1000 Hz]. SEMG signals were sampled at $2048 \mathrm{~Hz}$, converted in digital form by a 12 bit A/D converter and stored on disk. SEMG signals were digitally band-pass filtered (Butterworth, 4th order, 30-500 Hz). Furthermore, a notch filter (4th order Butterworth band stop with rejection width one $\mathrm{Hz}$ centered at two first harmonics of the power line frequency $(50 \mathrm{~Hz})$ ) was used in case of line interference.

Root mean square (RMS) was computed over $0.5 \mathrm{~s}$ non overlapping epochs. For all sites of EMG recording, EVA was computed over a logarithmic scale of the time level [ [ $\left.\begin{array}{lllll}3 & 5 & 9 & 17 & 33\end{array}\right] \mathrm{sec}$ and of the amplitude levels [ $\left[\begin{array}{llll}3 & 7 & 15 & 31\end{array}\right] \%$ of a reference contraction. Along the time and amplitude levels, the marginal distributions of EVA were computed by summing up its cells along the time and amplitude, beside that the EVA centroid location was extracted. Principle component analysis (PCA) was performed on both cases. Describing more than $80 \%$ of variance, first eight and four components were picked to be investigated for EVA marginal distributions and centroid respectively. Each component is composed of a linear transformation of EVA marginal distributions in the first case and EVA centroid in the second case. These values are known as "variables" in this context. As a rule of thumb, practical significant loadings in the transformation are larger than 0.5 , and in turn, the corresponding variable has significant contribution to that component [5].

\subsection{Statistics}

The practically significant PCA variables violated normality across the sessions (Shapiro-Wilk test). Therefore, Friedman test was performed to investigate the possible effect of the session on the variables. $P<0.05$ was considered significant.

\section{Results}

According the method described above, the significant variables were specified for designated components.

\subsection{EVA marginal distributions}

Eight designated components described $\sim 81 \%$ of total variance in the data set. Following variables contributed significantly to the designated components, all the significant variables were found along the amplitude levels:

- Component 1: Deltoid first level (below 3\%)

- Component 2: Serratus second level (3-7\%)

- Component 3: Descending first level (below $3 \%$ )

- Component 4: Serratus first level (below 3\%)

- Component 5: Ascending fifth level (above $31 \%)$

- Component 6: nothing above 0.5

- Component 7: Serratus third level (7-15\%)

- Component 8: Ascending third bin (7-15\%)

However, none of the variables changed significantly across the sessions. Figure 1 shows the variation of these variables across the sessions.

\subsection{EVA centroid}

Four designated components described $\sim 85 \%$ of total variance in the data set. Following variables contributed significantly to the designated components.

- Component 1: Deltoid along time levels

- Component 2: Ascending along time and amplitude levels

- Component 3: Descending along amplitude levels

- Component 4: Serratus along amplitude levels

However, none of the variables changed significantly across the sessions. Figure 2 shows the EVA centroid in the plane of the amplitude and time levels across the sessions.

\section{Discussion}

A novel approach was proposed to assess the EVA representation of physical exposure during computer work. Similar processing scheme has been utilized by [4] but the current method has the novelty to investigate the practically significant variables of PCA analysis and elaborate on the application of EVA among computer users.

PCA analysis of EVA marginal distribution indicated that practically significant variables are only along the amplitude levels and they are below $15 \%$ of reference contraction implying low contraction levels. This may support the studies which suggest that during computer work and low load tasks the attention has to be mainly paid to lower levels of contraction as they suggest to compute the relative rest time and 


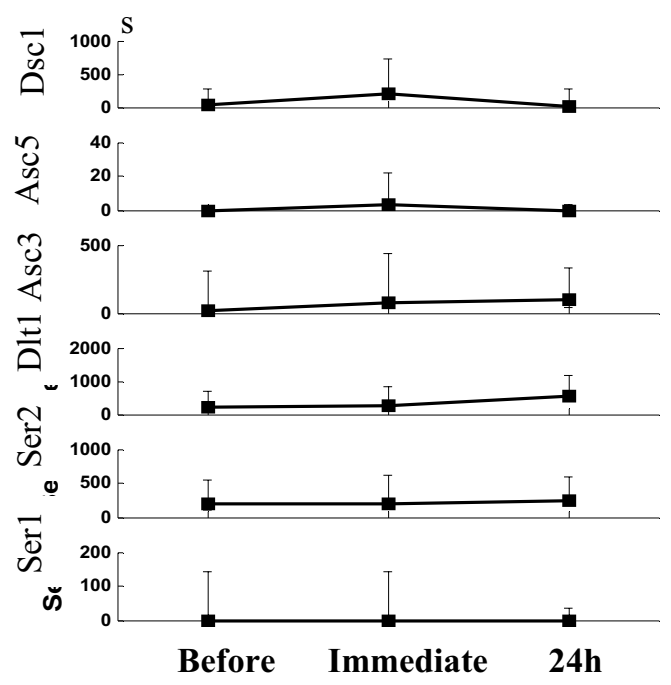

Fig 1. Median $\left[25^{\text {th }}-75^{\text {th }}\right.$ percentile] of practically significant variables from PCA analysis of EVA marginal distribution across the sessions. The Y-axis labels were abbreviated. Dsc1: First amplitude level of descending part of trapezius. Similarly, Asc, Dlt and Ser stand for ascending part of trapezius, deltoid anterior and serratus anterior respectively.

believe that more reliable results can be achieved compared with EMG amplitude [7].

Contrary to our hypothesis regarding a redistribution of load in response to altered sensory information, the results did not indicate a significant pattern implying the phenomenon. This is in accordance with our previous work on computer work and its interaction with pause type [16]. However, the recording time was shorter in that study, so longer recording time could have led to different results.

Generally, the assessment of a potential redistribution of shoulder muscle activity can be hard because of shoulder region complexity and the involvement of several synergistic muscles to provide the stability of shoulder girdle and glenohumeral joint [6]. This study only extracted the information form superficial muscles which can be studied using surface EMG, so the current results can not rule out any possible redistribution of load to deep muscles such as Rhomboid minor and major. These muscles have been suggested to take over the role of descending part of trapezius in response to biofeedback from upper part of trapezius [13].

In our previous study, combining linear and nonlinear features of surface EMG did not discriminate between states of muscles in response to altered peri- pheral stimuli [14]. Along with that inconclusive result, the current processing framework cannot in its present form be used to establish a biofeedback system. This may be in line with the studies which have not found an association between the level of muscle activity in trapezius and pain symptoms among office workers [18].

As a limitation of current study, we should note that the ratio between cases and variables is quite low in case of EVA marginal distributions PCA analysis. Besides, the limited subject pool does not provide a reliable statistical power for our test.

Otherwise, the trends of changes may have shown that for descending part of trapezius, the elapsed time in the first level of amplitude increased immediately after the exercise and then decreased to the baseline 24 hours after the exercise. This would be in line with our previous results during computer work where we showed a drop in EMG amplitude immediately after the exercise [14]. The non-significant results in the current study, however, might be due too coarse data reduction (few levels) or an undue dilution of data (many levels) in EVA analysis. This calls for developing methods to designate optimum levels of amplitude and time in EVA analysis. However, the challenge is to relate the selected levels of

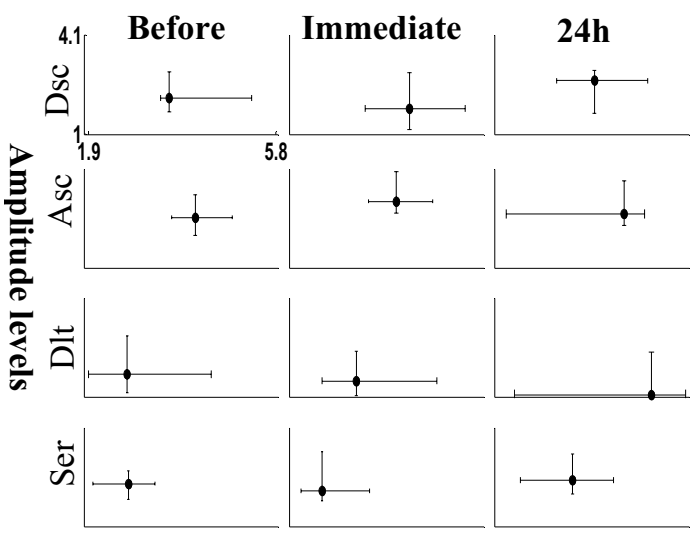

\section{Time levels}

Fig 2. EVA centroid (Median $\left[25^{\text {th }}-75^{\text {th }}\right.$ percentile $]$ ) in the plane of amplitude and time levels across the sessions. The Y-axis labels were abbreviated. Dsc, Asc, Dlt and Ser stand for descending and ascending part of trapezius, deltoid anterior and serratus anterior respectively.

EVA to corresponding exposure levels and repetitiveness which are probably associated with the risk factors of musculoskeletal disorders. 


\section{Conclusion}

In summary, the study suggests a modified approach to analyze the EVA representation of exposure pattern but could not verify any sign of redistri-

\section{References}

[1] A. K. Blangsted, K. Søgaard, H. Christensen and G. Sjøgaard, ,The effect of physical and psychosocial loads on the trapezius muscle activity during computer keying tasks and rest periods, Eur. J. Appl. Physiol., (2004) vol. 91, pp. 253258.

[2] D. Falla, D. Farina and T. Graven-Nielsen, ,Experimental muscle pain results in reorganization of coordination among trapezius muscle subdivisions during repetitive shoulder flexion, Exp Brain Res, (2007) vol. 178, pp. 385-393.

[3] J. A. Faulkner, S. V. Brooks and J. A. Opiteck, ,Injury to skeletal muscle fibers during contractions: conditions of occurrence and prevention, Phys. Ther., (1993) vol. 73, pp. 911.

[4] A. Fjellman-Wiklund, H. Grip, J. S. Karlsson and G. Sundelin, ,EMG trapezius muscle activity pattern in string players: Part I-is there variability in the playing technique? Int. J. Ind Ergonomics, (2004) vol. 33, pp. 347-356.

[5] J. F. Hair, R. E. Anderson, R. L. Tatham and W. C. Black, Multivariate Data Analysis. ,5th ed.Prentice Hall, Englewood Cliffs, NJ, 1998, pp. 730-87.

[6] D. H. Hawkes, O. Alizadehkhaiyat, A. C. Fisher, G. J. Kemp, M. M. Roebuck and S. P. Frostick, ,Normal shoulder muscular activation and co - ordination during a shoulder elevation task based on activities of daily living: An electromyographic study, Journal of Orthopaedic Research,

[7] H. J. Hermens and M. M. R. Vollenbroek-Hutten, ,Effects of electrode dislocation on electromyographic activity and relative rest time: Effectiveness of compensation by a normalisation procedure, Med Biol Eng Comput, (2004) vol. 42, pp. 502-508.

[8] P. Madeleine, F. Leclerc, L. Arendt-Nielsen, P. Ravier and D. Farina, ,Experimental muscle pain changes the spatial distribution of upper trapezius muscle activity during sustained contraction, Clin Neurophysiol, (2006) vol. 117, pp. 2436-2445.

[9] P. Madeleine, H. Nie and L. Arendt-Nielsen, ,Dynamic shoulder dynamometry: a way to develop delay onset muscle soreness in shoulder muscles, J. Biomech., (2006) vol. 39, pp. 184188.

[10] S. E. Mathiassen, J. Winkel and G. M. Hägg, ,Normalization of Surface EMG Amplitude from the Upper Trapezius Muscle in Ergonomic Studies, J Electromyogr Kinesiol, (1995) vol. 5, pp. 197-226.

[11]S. E. Mathiassen and J. Winkel, ,Quantifying variation in physical load using exposure-vs-time data, Ergonomics, (1991) vol. 34, pp. 1455-1468.

[12]H. Nie, A. Kawczynski, P. Madeleine and L. ArendtNielsen, ,Delayed onset muscle soreness in neck/shoulder muscles, Eur J Pain, (2005) vol. 9, pp. 653-660.

[13]G. Palmerud, H. Sporrong, P. Herberts and R. fors, ,Consequences of trapezius relaxation on the distribution of shoulder muscle forces: an electromyographic study, J. Electromyogr. Kinesiol., (1998) vol. 8, pp. 185-193.

[14]A. Samani, A. Kawczyński and P. Madeleine, "Supervised neuro-fuzzy biofeedback for computer users," in 15th Nordic- bution of load among other synergistic muscles. Further studies including deep muscles are required to investigate the redistribution of muscular load in response to DOMS during computer work.

Baltic Conference on Biomedical Engineering and Medical Physics (NBC 2011), 2011, pp. 33-36.

[15]A. Samani, A. Holtermann, K. Søgaard and P. Madeleine, Active pauses induces more variable electromyographic pattern of the trapezius muscle activity during computer work, J Electromyogr Kinesiol., (2009) vol. 19, pp. e430-437.

[16]A. Samani, A. Holtermann, K. Søgaard and P. Madeleine, ,Effects of eccentric exercise on trapezius electromyography during computer work with active and passive pauses, Clin. Biomech., (2009) vol. 24, pp. 619-625.

[17]P. Svensson and L. Arendt-Nielsen, ,Induction and Assessment of Experimental Muscle Pain, J Electromyogr Kinesiol, (1995) vol. 5, pp. 131-140.

[18] O. Vasseljen and R. H. Westgaard, ,A case-control study of trapezius muscle activity in office and manual workers with shoulder and neck pain and symptom-free controls, Int. Arch. Occup. Environ. Health, (1995) vol. 67, pp. 11-18.

[19]B. A. Winkelstein, ,Mechanisms of central sensitization, neuroimmunology \& injury biomechanics in persistent pain: implications for musculoskeletal disorders, J Electromyogr Kinesiol, (2004) vol. 14, pp. 87-93. 